\title{
Factors associated with the progression of gastric intestinal metaplasia: a multicenter, prospective cohort study
}

\section{다 $(9)$}

\section{Authors}

S. A. V. Nieuwenburg ${ }^{1}$, M. C. Mommersteeg*, 1, E. L. Eikenboom ${ }^{*, 1}$, B. Yu ${ }^{1}$, W. J. den Hollander ${ }^{2}$, I. Lisanne Holster ${ }^{1}$, Caroline M. den Hoed ${ }^{1}$, L. G Capelle ${ }^{3}$, Thjon J. Tang ${ }^{4}$, Marie-Paule Anten ${ }^{5}$, I. Prytz-Berset ${ }^{6}$, E. M. Witteman ${ }^{7}$, F. ter Borg $^{8}$, Jordy P. W. Burger ${ }^{9}$, Marco J. Bruno ${ }^{1}$, G. M. Fuhler ${ }^{1}$, Maikel P. Peppelenbosch ${ }^{1}$, Michael Doukas ${ }^{10}$, Ernst J. Kuipers ${ }^{1}$, Manon C.W. Spaander ${ }^{1}$

Institutions

1 Departments of Gastroenterology and Hepatology, Erasmus University Medical Centre, Rotterdam, The Netherlands

2 Leiden University Medical Centre, Leiden, The Netherlands

3 Meander Medical Centre, Amersfoort, the Netherlands

4 IJsselland Hospital, Capelle aan den IJssel, The Netherlands

5 Sint Franciscus Hospital, Rotterdam, The Netherlands

6 More and Romsdal Trust Ålesund, Ålesund, Norway

7 Canisius-Wilhelmina Hospital, Nijmegen, The Netherlands

8 Deventer Hospital, Deventer, The Netherlands

9 Department of Gastroenterology and Hepatology, Rijnstate, Arnhem, The Netherlands

10 Department of Pathology, Erasmus University Medical Centre, Rotterdam, The Netherlands

submitted 28.5.2020

accepted after revision

\section{Bibliography}

Endoscopy International Open 2021; 09: E297-E305

DOI 10.1055/a-1314-6626

ISSN 2364-3722

(C) 2021. The Author(s).

This is an open access article published by Thieme under the terms of the Creative Commons Attribution-NonDerivative-NonCommercial License, permitting copying and reproduction so long as the original work is given appropriate credit. Contents may not be used for commecial purposes, or adapted, remixed, transformed or built upon. (https://creativecommons.org/licenses/by-nc-nd/4.0/)

Georg Thieme Verlag KG, Rüdigerstraße 14,

70469 Stuttgart, Germany

Corresponding author

Manon C.W. Spaander, MD, PhD, Associate professor, Department of Gastroenterology and Hepatology (room Na-610), Erasmus MC University Medical Center, Doctor Molewaterplein 40, 3015 GD Rotterdam, The Netherlands
Fax: +31-0-10 7035172

v.spaander@erasmusmc.nl

$\bigoplus$ Supplementary material is available under https://doi.org/10.1055/a-1314-6626

\section{ABSTRACT}

Background and study aims Gastric cancer (GC) is usually preceded by premalignant gastric lesions (GPLs) such as gastric intestinal metaplasia (GIM). Information on risk factors associated with neoplastic progression of GIM are scarce. This study aimed to identify predictors for progression of GIM in areas with low GC incidence.

Patients and methods The Progression and Regression of Precancerous Gastric Lesions (PROREGAL) study includes patients with GPL. Patients underwent at least two upper endoscopies with random biopsy sampling. Progression of GIM means an increase in severity according to OLGIM (operative link on gastric intestinal metaplasia) during followup (FU). Family history and lifestyle factors were determined through questionnaires. Serum Helicobacter pylori infection, pepsinogens (PG), gastrin-17 and GC-associated single nucleotide polymorphisms (SNPs) were determined. Cox regression was performed for risk analysis and a chisquared test for analysis of single nucleotide polymorphisms.

Results Three hundred and eight patients (median age at inclusion 61 years, interquartile range (IQR: 17; male 48.4\%; median FU 48 months, IQR: 24) were included. During FU, 116 patients (37.7\%) showed progression of IM and six patients $(1.9 \%)$ developed high-grade dysplasia or GC. The minor allele (C) on TLR4 (rs11536889) was inversely associated with progression of GIM (OR 0.6 ; $95 \% \mathrm{Cl} 0.4-$ 1.0). Family history (HR $1.5 ; 95 \% \mathrm{Cl} 0.9-2.4)$ and smoking (HR 1.6; $95 \% \mathrm{Cl} 0.9-2.7$ ) showed trends towards progression of GIM. Alcohol use, body mass index, history of H. pylori infection, and serological markers were not associated with progression.

\footnotetext{
* These authors contributed equally as second authors
} 
Conclusions Family history and smoking appear to be related to an increased risk of GIM progression in low GC incidence countries. TLR4 (rs11536889) showed a significant inverse association, suggesting that genetic information may play a role in GIM progression.

\section{Introduction}

Gastric cancer (GC) of the intestinal type is often initiated by chronic Helicobacter pylori infection through gastritis eventually leading to the development of gastric premalignant lesions (GPLs). These in particular include atrophic gastritis (AG), gastric intestinal metaplasia (GIM), and dysplasia [1, 2]. These premalignant lesions make GC suitable for screening and surveillance. In high endemic GC regions such as Asia, populationbased screening for GC has been implemented in certain regions [3]. Due to low prevalence rates of GC, screening programs are not effective in low endemic areas such as the Northern European countries. These regions rely on surveillance programs of at-risk individuals with GPL identified during routine endoscopy.

The balance between burden and benefit of such endoscopic surveillance in low endemic regions is still under debate. While US guidelines do not recommend endoscopic surveillance, European guidelines recommend surveillance dependent on the extent and severity of GPL [4-7]. The American Gastroenterological Association (AGA) recently published clinical practice guidelines on the management of GIM. The AGA suggests against routine endoscopic surveillance for patients with GIM unless a higher risk of GC is the case (i.e. incomplete GIM, extensive GIM, a family history of GC or of Asian heritage) [8]. While European guidelines do opt for endoscopic surveillance, they recognize that a more individualised surveillance strategy distinct based on risk factors for disease progression is needed and will maximise the yield and reduce the burden of surveillance endoscopies. The recently updated European guideline takes risk factors such as a positive family history for gastric cancer into account [9].

Evidence suggests that patients who have a first-degree relative with gastric cancer (diffuse type [hereditary] gastric cancer excluded) have an increased risk for neoplastic progression compared to GIM patients who have no first-degree relative with gastric cancer [10-12]. Single nucleotide polymorphisms (SNPs) are common genetic variants among individuals and are frequently inherited within families. SNPs are increasingly being studied in association with $\mathrm{H}$. pylori infection and gastric cancer. SNPs that have been associated with non-cardia gastric carcinogenesis are located in toll like receptor 4 (TLR4) rs11536889, toll like receptor 1 (TLR1) rs28393318 (both having a signalling function in initiating immune responses), autophagy 16 like 1 (ATG16L1) rs2241880, and neutrophil cytosolic factor 4 (NCF4) rs482154 genes (playing a part in activity of granulocytes) [13-15]. Besides genetic factors, smoking, alcohol use and increased BMI have also been identified as possible risk factors for neoplastic progression of GPL, in particular in high-risk populations $[16,17]$. Serum pepsinogen (ratios) and gastrin levels are markers for current presence of an atrophic stomach [18]. Pepsinogen I/II ratio is increased, and gastrin level decreased in case of gastric atrophy [19]. However, it is not known if these serological markers have a predictive value for progression of intestinal metaplasia over time.

All in all, information on risk factors of neoplastic progression of GIM is limited and they have not been tested systematically in a prospective cohort of premalignant gastric lesion in patients living in a low GC incidence country. Many patients are followed intensively without risk stratification, causing a significant burden to patients and health care systems. From 2009, a prospective cohort of over 300 patients with premalignant gastric lesions was initiated in the Netherlands and Norway. This study aims to reveal potential risk factors associated with the progression of GIM, including analyses on SNPs and serological markers, in a country with low GC risk.

\section{Patients and methods}

\section{Patient selection}

The PROREGAL study (Progression and Regression of precancerous Gastric Lesions) was initiated in 2009 and is an ongoing prospective cohort study in six hospitals (one academic, five regional) in the Netherlands and one regional hospital in Norway. The study design has been described previously [20]. In short, patients over 18 years of age and with atrophic gastritis, gastric intestinal metaplasia and/or dysplasia are eligible for inclusion. Patients are excluded from participation if they have: 1) previously undergone upper gastrointestinal surgery, 2) a previous diagnosis of gastric carcinoma, or any other malignancy not being in remission, 3) severe comorbidity limiting their expected survival to less than 2 years, 4) portal hypertension, or 5) a proven $\mathrm{CDH} 1$ mutation. Eligible patients are included after written informed consent. The study protocol was approved by the Erasmus MC Institutional Review Board (MEC-2009-090).

\section{Baseline data collection}

Information on lifestyle factors, medical history, medication use and family history of gastric cancer were obtained through questionnaires. Pepsinogens-I/II (PG-I/II), gastrin-17 and H. pylori status blood samples were collected at baseline. Any active $H$. pylori infections were eradicated and confirmed afterwards.

\section{Endoscopy procedures}

All patients underwent at least one surveillance endoscopy after the index endoscopy (t0). First surveillance endoscopy (t1) was performed one year after the index endoscopy and the second surveillance endoscopy (t2) 3 years after the index endoscopy, except if low-grade dysplasia (within 1 year) or high-grade dysplasia (within 6 months) was present. Further 
follow-up surveillance endoscopies were decided according to the Management of epithelial precancerous conditions and lesions in the stomach (MAPS) guideline recommendations that became available during the study [4]. Every gastric lesion found at endoscopy was reported. Gastric biopsies were sampled in a standardised manner at every endoscopy. This included biopsies from any visible lesion and twelve random biopsies from five areas in the stomach: four quadrant biopsies of the antrum, two biopsies from the incisura, two from the lesser curvature, two from the greater curvature and two from the cardia (Supplementary Fig. 1).

\section{Pathology}

The biopsy specimens were fixed in buffered formalin and embedded in paraffin and then assessed by pathologists from the participating hospitals. The presence and grade of the different stages of GGIM were classified according to the OLGIM (Operative Link of Gastric Intestinal Metaplasia) classification. The scoring of lesions is divided into mild-moderate-severe IM in both antrum and corpus, providing a score between 0-IV with IV having the highest risk of gastric cancer development [21].

\section{Enzyme linked immunosorbent assay}

Serum was collected from patients at the first surveillance endoscopy and was aliquoted and stored at $-80^{\circ} \mathrm{C}$ until analysis. Ennzyme linked immunosorbent assay (ELISA) for $H$. pylori $\mathrm{IgG}$, gastrin and pepsinogen I/II was performed according to manufacturer's protocol (Gastropanel, Biohit oyi, Finland). In short, precoated plates were incubated with patient sera or standardized controls. Plates were washed and subsequently incubated with the conjugated antibody. After another wash step the substrate was added after which the readout could be performed at a frequency of $450 \mathrm{~nm}$. Optical density (OD) measurement and 8 subsequent quantification of plates was performed with the infinite 200 pro ELISA reader (TECAN, Mannedorf, Switzerland).

\section{Identification of SNPs}

Several SNPs associated in the past with $H$. pylori infection and an increased risk of gastric cancer were selected [22-25]. We first tested if there was a difference in the minor allele frequency (MAF) of the selected SNPs between the PROREGAL cohort and the general population. For this purpose, we compared the MAF of the PROREGAL cohort with the MAF found in the Rotterdam Study 1 cohort (RS1). The Rotterdam Study is a prospective cohort study of healthy persons living in a well-defined district in Rotterdam (Ommoord), more details on this study are described elsewhere [26]. The cohort reflects the general Dutch population. In short, it comprises almost 6,500 healthy participants aged between $45-75$ years. Participants are followed throughout life every 3-4 years with emphasis on collecting bio specimens that enable molecular and genetic analysis.

To determine a significant difference between the SNPs in the PROREGAL cohort versus the general population (RS1) a chi squared test was performed. The ATG16L1 (rs2241880) SNP MAF was 0.465 in the RS1 cohort versus 0.528 in the PROREGAL cohort $(P=0.016)$. The NCF4 ( $r$ 482154) SNP MAF was 0.292 in the RS1 cohort versus 0.311 in the PROREGAL cohort $(P=$ 0.530). The TLR1 (rs28393318) SNP MAF was 0.266 in the RS1 cohort versus 0.374 in the PROREGAL cohort $(P>0.001)$. The TLR4 (rs11536889) SNP MAF was 0.147 in the RS1 cohort versus 0.192 in the PROREGAL cohort $(P=0.048)$. Based on these results we included rs2241880, rs28393318 and rs11536889 SNPs for the current study.

\section{DNA isolation and SNP identification in the PROREGAL cohort}

At baseline blood was collected from each patient. DNA was isolated using the Kleargene XL blood DNA extraction kit (LGC limited, Teddington, UK). Quantity and quality of isolated DNA was measured on the nanodrop and DNA samples were normalized to $10 \mathrm{ng} / \mathrm{ul}$. SNPs were determined using Polymerase Chain Reaction-Restriction Fragment Length Polymorphism (PCRRFLP). In short DNA fragments were amplified using regular PCR for 35 cycles. After quality control by performing gel electrophoresis on $2 \%$ Tris-Borate-EDTA (TBE) agarose gel, samples that provided a well-defined band are digested overnight using a restriction enzyme that specifically digest one allele of the appropriate SNP (Supplementary Table 1). These samples were then used in TBE gel electrophoresis and presence of the SNP in one or two alleles was determined by identifying the number of bands: one band for homozygously undigested, two bands for homozygously digested, three bands for heterozygous patients.

\section{Statistical analyses}

Baseline characteristics are presented as mean with standard deviation (SD) or median with interquartile range (IQR). Categorical variables were reported as percentages. Progression of GIM was defined as progression of the OLGIM classification at any time point between follow-up (FU) endoscopies. Potential risk factors (RF) for progression were analysed by Cox-regression with a two-sided significance level of 0.05 and providing hazard ratios (HR) $95 \% \mathrm{Cl}$. To further substantiate the genetic role on one's progression risk, interdependence of SNP distributions for progressors and non-progressors was calculated using the chi squared test and providing odds ratio (OR) with $95 \%$ confidence intervals $(95 \% \mathrm{Cl})$. Analyses were performed in IBM SPSS v.24. Figures were drafted in R V.3.4.2.

\section{Sample size and selection of predictors}

To avoid overfitting of the Cox regression model we have used an event per variable (EPV) of $>10$ with a central limit theorem of 10 cases as generally proposed in sample size considerations for proportional hazards analysis [27]. This entails that for every ten cases of progression of GIM one predictor was added to the cox model starting from the $10^{\text {th }}$ case. The current study contains 81 cases of progression with complete data. This translates into the inclusion of seven predictors ( 81 cases minus 10 cases as being the central limit of theorem, adding 1 variable per 10 cases) in our Cox regression model.

We pre-selected possible predictors that can be determined prior to one's first upper endoscopy, based on available litera- 


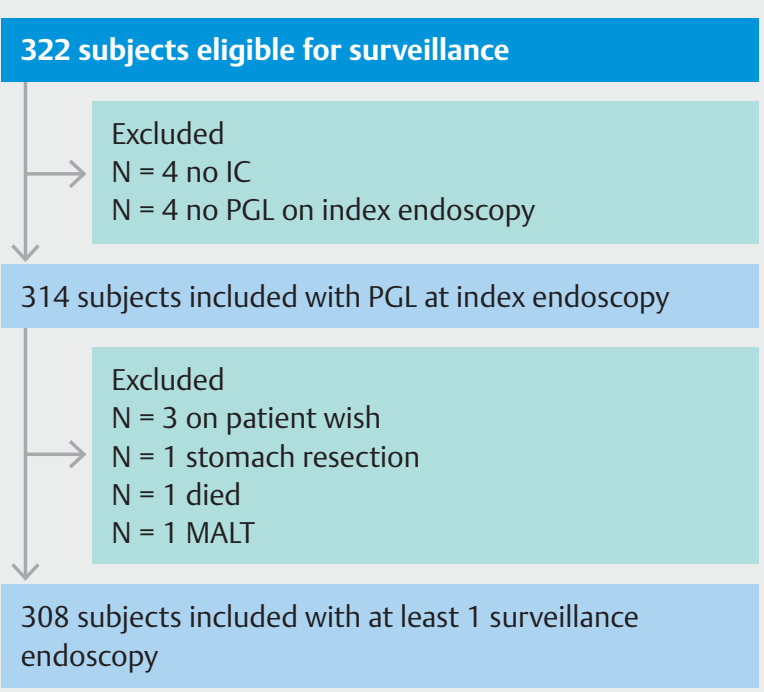

- Fig. 1 Flowchart of the included subjects. IC, informed consent; PGL, premalignant gastric lesion; MALT, mucosa-associated lymphoid tissue.

ture and guidelines. Previously, our study group and many others have shown a correlation between OLGIM stage and values of the serological markers pepsinogen I, pepsinogen II, and gastrin-17 [20,28]. It is therefore that we included these serological markers in the current study to evaluate if these factors might also be associated with the progression of GIM over time.

Further, family history has been an increased focus of research and was recently added as a risk factor in the updated surveillance guidelines [9]. However, it is still rated as "low quality evidence" for which prospective data is needed.

Several trials have been performed on the effects of $H$. pylori eradication and the progression to gastric cancer. The benefits of $H$. pylori eradication were mostly seen in patients without premalignant gastric lesions at baseline $[29,30]$. We have added history of an (confirmed eradicated) $H$. pylori infection to evaluate if this might affect progression of GIM over time. Lifestyle factors such as smoking, use of alcohol and body mass index (BMI) remain understudied with controversial results and mainly focus on the occurrence of gastric cancer. Associations with the progression of premalignant lesions might just be as important [31-34].

\section{Results}

\section{Baseline characteristics}

A total of 308 patients were included ( $\downarrow$ Fig. 1). Their median age was 61 years (IQR: 17) and $48.4 \%$ were male. Baseline characteristics of the study population are shown in $>$ Table 1 . Median follow-up (FU) time was 48 months (IQR 24), with a median of three endoscopies (IQR: 1) performed per patient. One hundred and sixteen patients (38.0\%) showed progression of OLGIM stage, two (0.6\%) patients showed progression to highgrade dysplasia, and four patients $(1.3 \%)$ developed gastric
- Table 1 Baseline characteristics of the PROREGAL cohort.

\begin{tabular}{|c|c|c|}
\hline Baseline characteristics & $\begin{array}{l}\text { PROREGAL } \\
\text { cohort }\end{array}$ & $\begin{array}{l}\text { Available } \\
\text { data } \\
\mathrm{N} \text { (\% from } \\
\text { total) }\end{array}$ \\
\hline Male n (\%) & $148(48)$ & $308(100)$ \\
\hline Age at baseline (years) median (IQR) & $61(17)$ & $308(100)$ \\
\hline Ethnicity (Caucasian), n (\%) & $242(79)$ & $308(100)$ \\
\hline Follow up (months) median (IQR) & $48(24)$ & $308(100)$ \\
\hline OLGIM stage at baseline n (\%) & & $308(100)$ \\
\hline 0 & $54(21)$ & \\
\hline । & $83(32)$ & \\
\hline II & $67(26)$ & \\
\hline III & $42(16)$ & \\
\hline IV & $10(4)$ & \\
\hline Progression of IM n (\%) & $116(38)$ & $308(100)$ \\
\hline Progression to HGD n (\%) & $2(0.6)$ & $308(100)$ \\
\hline Progression to gastric cancer, n (\%) & $4(1.3)$ & $308(100)$ \\
\hline History of $H$. pylori infection, $\mathrm{n}(\%)$ & $148(62)$ & $237(77)$ \\
\hline Pepsinogen I ( $\mu \mathrm{g} / \mathrm{L})$ mean $(\mathrm{SD})$ & $128(99)$ & $301(98)$ \\
\hline Pepsinogen II $(\mu \mathrm{g} / \mathrm{L})$ mean $(\mathrm{SD})$ & $18(13)$ & $303(98)$ \\
\hline Pepsinogen $1 / I I(\mu \mathrm{g} / \mathrm{L})$ mean $(\mathrm{SD})$ & $9(11)$ & $301(98)$ \\
\hline Gastrin-17 (pmol/L) mean (SD) & $19(25)$ & $296(96)$ \\
\hline Smoking status (ever), n (\%) & $151(60)$ & $250(81)$ \\
\hline Alcohol use (yes), n (\%) & $132(53)$ & $249(81)$ \\
\hline Family history of gastric cancer, $\mathrm{n}$ (\%) & $76(29)$ & $262(85)$ \\
\hline First degree & $50(16)$ & \\
\hline Second degree & $26(8)$ & \\
\hline \multicolumn{3}{|c|}{$\begin{array}{l}\text { PROREGAL, Progression and Regression of Precancerous Gastric Lesions; } \\
\text { IQR, interquartile range; OLGIM, Operative Link for Gastritis Assessment; } \\
\text { IM, intestinal metaplasia; HGD, high-grade dysplasia. }\end{array}$} \\
\hline
\end{tabular}

cancer. The distribution of progression within specific OLGIM stages of these progressors is shown in $\mathbf{F i g .} \mathbf{2}$.

\section{Risk factors}

Smoking (HR 1.6; $95 \% \mathrm{Cl} 0.9-2.7, P=0.079$ ) and having a family member (first- and/or second-degree) with gastric cancer (HR 1.5; $95 \% \mathrm{Cl} 0.9-2.4, P=0.076)$ was associated with an increased risk of progression of GIM, but statistically non-significant ( $\triangleright$ Fig. 3). Serum PG I, PG II and their PG I/II ratio (HR 1.0; $95 \% \mathrm{Cl} 0.9-1.1, P=0.420)$ as well as serum gastrin-17 (HR 1.0; $95 \% \mathrm{Cl} 0.9-1.1, P=0.854)$ did not significantly correlate with the risk of GIM progression. Also, history of an $\mathrm{H}$. pylori infection (HR 1.1; 95\%Cl 0.6-1.7, $P=0.953)$, alcohol use (HR 0.7; $95 \% \mathrm{Cl}$ $0.4-1.1, P=0.103)$, and BMI (HR 1.0; $95 \% \mathrm{Cl} 0.9-1.1, P=0.947)$ showed no association with progression of GIM. 


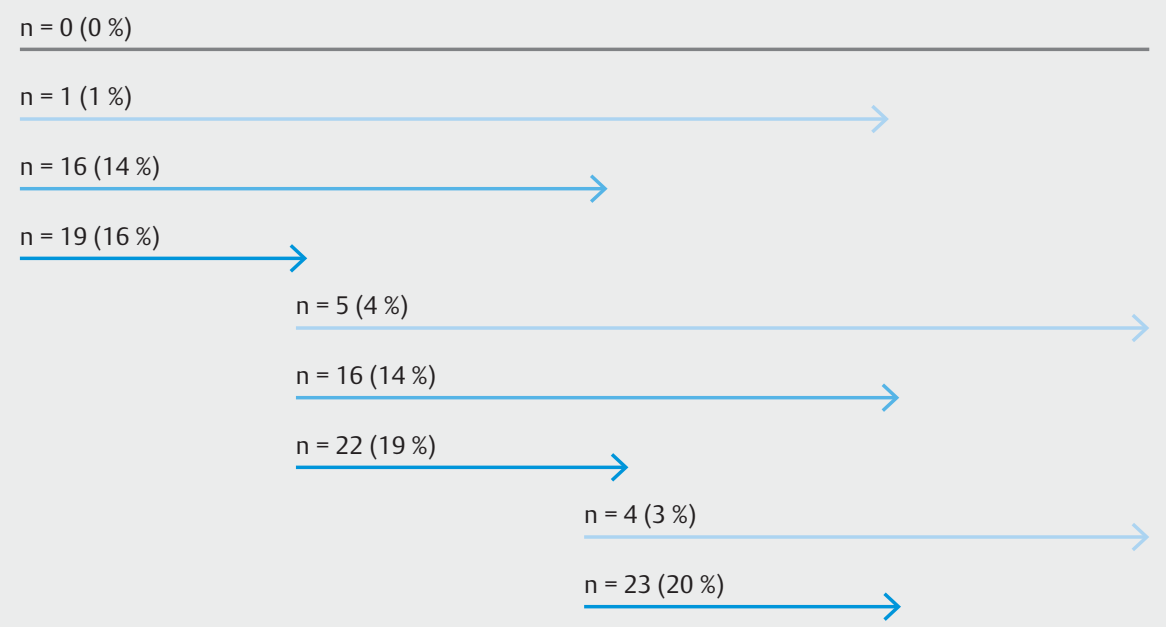

- Fig. 2 Percentages of progressors per OLGIM stage at baseline and maximal OLGIM stage at the first time point of progression during follow up.

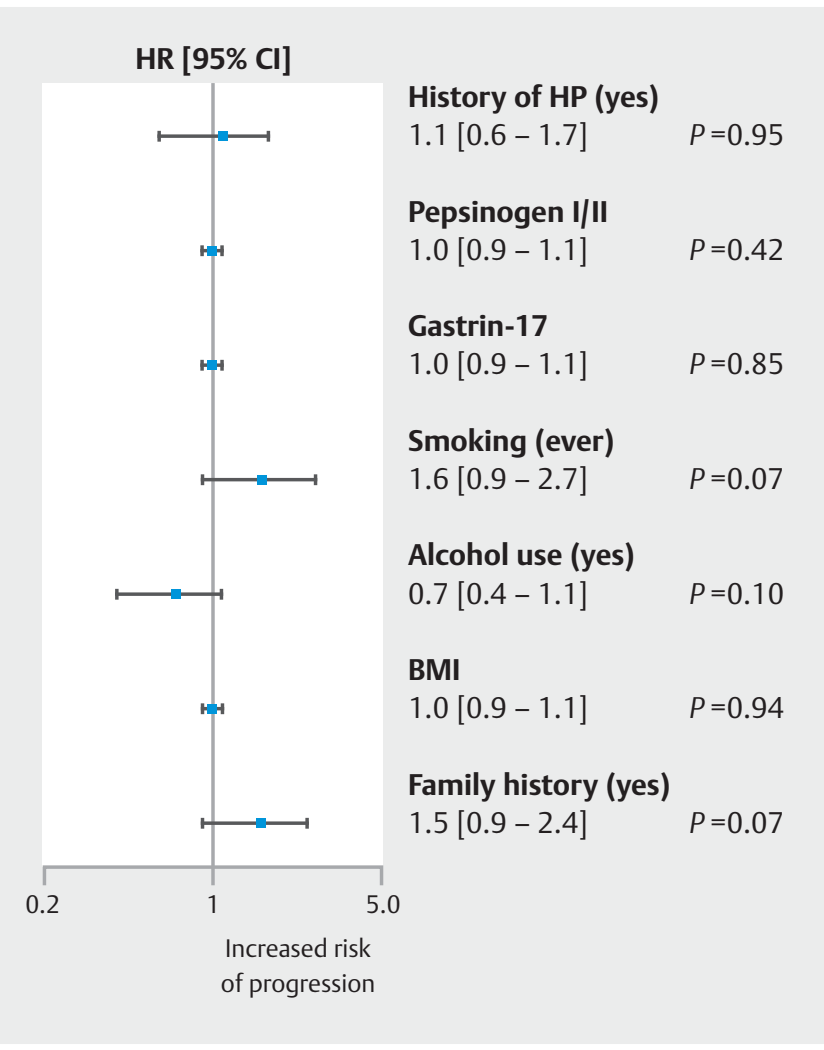

Fig. 3 Analysis of risk factors associated with progression of intestinal metaplasia. $\mathrm{BMI}$, body mass index; $\mathrm{Cl}$, confidence interval; Hp, Helicobacter pylori; HR, hazard ratio.

\section{Family history of gastric cancer}

Information on family history was available for 266 subjects (86\%). Fifty subjects had a first-degree relative with gastric cancer (of whom $48.0 \%$ showed progression of IM), 26 had a second-degree relative with gastric cancer (of whom 50.0\% showed progression of IM), and 190 did not have a family history of gastric cancer (of whom $36.3 \%$ showed progression of IM) ( Fig. 4).

\section{Single nucleotide polymorphisms}

The genotype distribution of the SNPs within our cohort is represented in $\boldsymbol{\nabla}$ Table 2 . Also, this demonstrates the minor allele frequencies for all variants between the non-progressor and progressor groups. The minor allele (C) on the TLR4 gene (rs11536889) was inversely associated with the progression of GIM showing an odds ratio (OR) of $0.6(P=0.042)$.

\section{Discussion}

To our knowledge, this is the first study that prospectively assessed multiple risk factors, including SNP analysis, for progression of gastric intestinal metaplasia in a population with a low gastric cancer incidence. SNP analysis showed that the minor allele (C) on TLR4 (rs11536889) was negatively associated with progression of GIM. This result suggests genetic information may play a role in GIM progression. Possible risk factors that were previously identified in high-risk GC populations were not predictive for progression in our low-risk population. However, a positive family history of gastric cancer and smoking status might be associated with an increased risk of progression.

Lifestyle factors such as the use of alcohol and smoking were previously studied in association with gastric cancer. A large meta-analysis including 59 studies showed a correlation be- 
308 subjects with a GPL from the proregal cohort

\section{$\downarrow$}

266 subjects with available information on family history

\begin{tabular}{|c|c|c|}
\hline & $\downarrow$ & $\downarrow$ \\
\hline 190 subjects & 50 subjects & 26 subjects SD \\
with no family & with FD relative & relative with \\
history of GC & with GC & GC \\
\hline$\downarrow$ & $\downarrow$ & $\downarrow$ \\
$36.3 \%$ & $48.0 \%$ & $50.0 \%$ \\
progression of & progression of & progression of \\
IM & IM & IM \\
\hline
\end{tabular}

- Fig.4 Flowchart of available information on family history. Subjects of the PROREGAL cohort with known gastric premalignant lesions and the proportion of subjects with a positive family history. FD, first-degree relative; GC, gastric cancer; IM, intestinal metaplasia; SD, second-degree relative.

tween heavy drinking ( $>4$ drinks/day) and progression to noncardia gastric cancer with a relative risk (RR) of $1.39(95 \% \mathrm{Cl}$ 1.14-1.69) among non-Asian studies and $0.90(95 \% \mathrm{Cl} 0.65-$ 1.25) among Asian studies [34]. In our study we did not find any correlation between alcohol consumption and progression of IM. However, our data did not allow for discrimination between amounts of consumption, which might neglect the influence of heavy drinking in our cohort.
A large Korean cohort study including almost 200,000 subjects found smoking as an independent risk factor for the development of IM by measuring urinary cotinine levels. Heavy smoking (i. e. nicotine level $>500 \mathrm{ng} / \mathrm{mL}$ ) corresponded to an hazard ratio (HR) of $1.54(95 \% \mathrm{Cl} 1.44-1.64)$ for men, and 1.57 ( $95 \% \mathrm{Cl} 1.07-2.30)$ for women [35]. In a study from a low risk area, a HR of 1.13 (95\% Cl 1.00-1.27, $P=0.05$ ) was found for the progression to gastric cancer in male smokers with normal serum PGI levels [36]. A recent meta-analysis pooled the published studies on smoking and alcohol use and their relation with the presence of GIM [37]. By pooling these results ( $n=$ 7971 subjects in eight studies) the Relative Risk (RR) of ever or former smoking versus never smoked and the presence of GIM was 1.57 (95\% Cl 1.24-1.98). For ever or former alcohol use versus no alcohol use ( $\mathrm{n}=6775$ subjects in five studies) RR was 1.29 (95\% 1.12-1.50). Although not significant, we found similar trends for the risk of progression of GIM in smokers.

Serum markers such as gastrin-17 and pepsinogen I and II are well correlated with the presence and severity of premalignant lesions $[18,20,38]$. This study aimed to evaluate if serological markers at baseline might have a predictive value for future progression of IM. This would substantiate proper risk stratification at initial diagnosis. However, our results did not show any significant associations between serology levels at baseline and progression of GIM during follow up. It is still to be tested if longitudinal data assessment of serological markers during every FU endoscopy might be of value.

In both the American Gastroenterological Association (AGA) and European guidelines, routine surveillance endoscopies are not recommended for all patients with GPL. Both guidelines advise a more individualized strategy where accurate risk stratification is the key. Both emphasize the importance of having a positive family history of gastric cancer as being a risk factor. Al-

- Table 2 Summary of the genotypes associated with progression in the PROREGAL cohort and comparison of the minor allele frequencies (MAF) in the non-progression and progression groups.

\begin{tabular}{|c|c|c|c|c|c|}
\hline & Genotype & Non-progression (\%) & Progression (\%) & OR (95\%Cl) & $P$ value \\
\hline \multirow[t]{3}{*}{ ATG16L1 } & AA & $30(18.6 \%)$ & $23(21.3 \%)$ & & \\
\hline & AG & $91(56.5 \%)$ & $57(52.8 \%)$ & & \\
\hline & GG & $40(24.8 \%)$ & $28(25.9 \%)$ & & \\
\hline MAF & & 0.469 & 0.477 & $1.0(0.8-1.4)$ & 0.808 \\
\hline \multirow[t]{3}{*}{ TLR1 } & AA & $57(35.4 \%)$ & $36(33.6 \%)$ & & \\
\hline & GA & $92(57.1 \%)$ & $59(55.1 \%)$ & & \\
\hline & GG & $12(7.5 \%)$ & $12(11.2 \%)$ & & \\
\hline MAF & & 0.360 & 0.388 & $1.1(0.8-1.5)$ & 0.572 \\
\hline \multirow[t]{3}{*}{ TLR4 } & GG & 95 (59.4\%) & 80 (74.1\%) & & \\
\hline & GC & $56(35.0 \%)$ & $25(23.1 \%)$ & & \\
\hline & CC & $9(5.6 \%)$ & $3(2.8 \%)$ & & \\
\hline MAF & & 0.231 & 0.144 & $0.6(0.4-1.0)$ & 0.042 \\
\hline
\end{tabular}


though not significant, the recent meta-analysis showed that a positive family history was associated with the presence of GIM (RR1.46, 95\%Cl 0.97-2.21) [37]. Both the AGA guidelines as well as the updated MAPS guidelines recommend providing more intensive surveillance in this at-risk population $[8,9]$. Our results indeed point in the direction of an increased risk of progression of GIM in case of a positive family history. The current study helps strengthen the currently available literature by further elucidating these risk factors, as well as showing that familial predisposition might also contribute to the course of disease.

These results are in line with previous literature. A prospective cohort study from Italy also showed that neoplastic progression in AG patients was two-fold more frequent among patients with a first degree relative with gastric cancer compared to patients with a negative family history $(23.5 \%$ vs. $12.6 \%, P=$ 0.4867 ) [39]. In a retrospective study from the United States, over 900 subjects with IM were included. Of these, 25 subjects progressed to gastric cancer, with family history being a significant risk factor (incidence rate ratio, 8.87; $95 \% \mathrm{Cl} 1.5-23.5 ; P=$ 0.012) [40].

The ATG16L1 SNP rs2241880 previously has been associated with gastric cancer [25]. In the PROREGAL cohort, variation at rs2241880 was associated with the presence of intestinal metaplasia when compared to a general population control group. Rs2241880 is a functional SNP that results in an amino acid change of threonine to alanine at position 300 in the ATG16L1 protein, which in turn causes impaired autophagy [41]. Increasing evidence links the autophagy pathway to $H$. pylori pathogenesis [42]. Variation in the TLR1 gene at rs28393318 is in linkage disequilibrium with the functional SNP rs4833095 which has previously been negatively associated with $\mathrm{H}$. pylori serology and positively associated with gastric cancer [43, 44]. In our study, rs28393318 was also associated with the presence of gastric premalignant lesions. Although the consequences of this SNP remain largely unclear, it is biologically plausible that innate immune signaling through TLR1 may contribute to the inflammatory environment in which gastric premalignant lesions may occur.

Of the analyzed SNPs, only variation at TLR4 (rs11536889) showed a significant inverse association with the progression of IM. Just as with TLR1, TLR4 is a pattern recognition receptor and can initiate the innate immune response in the host colonized with $H$. pylori. This SNP might thus affect the intensity of the host response against $H$. pylori and thus modify the severity of chronic $H$. pylori gastritis [13]. The association of rs11536889 with IM and the inverse relationship with progression of IM may seem contradictory. However, several explanations can be found for these conflicting results. First, all previous studies that found an increased risk of gastric cancer associated with rs11536889 were restricted to patients of Chinese descent $[13,45]$. Second, the highest risk that could be found was in individuals that, aside from the variation at rs11536889, were also infected with $H$. pylori. This is also biologically plausible since $T L R$ signalling is important in the early response to $H$. pylori infection. Several studies suggest that over the long term, modest regression of gastric premalignant lesions may be ex- pected after eradication of $H$. pylori $[46,47]$. If the association of this TLR4 SNP with gastric cancer is co-dependent on $\mathrm{H}$. pylori infection, one might expect some regeneration or stabilization when $\mathrm{H}$. pylori has been eradicated.

Our study also has some limitations. First, our median follow-up period is 4 years. To draw firm conclusions on the course of disease with concomitant risk factors, a longer follow-up period is needed. Second, lifestyle risk factors and family history were obtained through questionnaires, making that information subject to patient interpretation. Third, due to a limited number of cases we were not able to add more potential risk factors. Because the PROREGAL study is an ongoing prospective study that will be continued and expanded in the future, more long-term data with larger sample size are awaited. Fourth, the association between smoking and a positive family history was not statistically significant, with a $P=0.07$; it may, however, point in the direction of an indicated effect. Furthermore, our primary endpoint was progression of OLGIM stage instead of progression to gastric cancer because of the small number of neoplastic lesions. Location, therefore, could not to be added as independent factor in the Cox model. For low-risk regions, it is just as important to focus on the identification of progression of premalignant lesions as to stop further surveillance in patients with a very low risk of gastric cancer development.

\section{Conclusion}

In conclusion, this multicenter, prospective cohort study on the surveillance of gastric premalignant lesions in an area with a low gastric cancer incidence shows that both a positive family history of gastric cancer and a history of smoking are indicated to have an effect on the progression of GPL. This study further substantiates the possible underlying role of SNPs in (non-)progression of GPL, suggesting that genetic information may play a role in the risk stratification of patients with GIM.

\section{Acknowledgments}

The authors would like to acknowledge Dr. Linda Broer and Prof. Dr. André Uitterlinden for providing data from the Rotterdam Study.

\section{Competing interests}

During the conduct of the study, Dr. Bruno received grants from Boston Scientific, personal fees from Boston Scientific, grants from Cook Medical, personal fees from Cook Medical, grants from Pentax Medical, personal fees from Pentax Medical, grants from $3 \mathrm{M}$, personal fees from $3 \mathrm{M}$, grants from Mylan, and personal fees from Mylan. Dr Spaander received research support from Medtronic and Boston Scientific. 


\section{References}

[1] IARC Working Group. Schistosomes, liver flukes and Helicobacter pylori. IARC Working Group on the Evaluation of Carcinogenic Risks to Humans. Lyon, 7-14 June 1994. IARC Monogr Eval Carcinog Risks Hum 1994; 61: 1-241

[2] Plummer M, Franceschi S, Vignat J et al. Global burden of gastric cancer attributable to Helicobacter pylori. Int J Cancer 2015; 136: 487490

[3] Fock KM, Talley N, Moayyedi P et al. Asia-Pacific consensus guidelines on gastric cancer prevention. J Gastroenterol Hepatol 2008; 23: 351365

[4] Dinis-Ribeiro M, Areia M, de Vries AC et al. Management of precancerous conditions and lesions in the stomach (MAPS): guideline from the European Society of Gastrointestinal Endoscopy (ESGE), European Helicobacter Study Group (EHSG), European Society of Pathology (ESP), and the Sociedade Portuguesa de Endoscopia Digestiva (SPED). Endoscopy 2012; 44: 74-94

[5] Trieu JA, Bilal M, Saraireh $\mathrm{H}$ et al. Update on the diagnosis and management of gastric intestinal metaplasia in the USA. Dig Dis Sci 2019; 64: 1079-1088

[6] Banks M, Graham D, Jansen M et al. British Society of Gastroenterology guidelines on the diagnosis and management of patients at risk of gastric adenocarcinoma. Gut 2019; 68: 1545-1575

[7] Evans JA, Chandrasekhara V. ASGE Standard of Practice Committee. et al. The role of endoscopy in the management of premalignant and malignant conditions of the stomach. Gastrointest Endosc 2015; 82: $1-8$

[8] Gupta S, Li D, El Serag HB et al. AGA Clinical Practice Guidelines on Management of Gastric Intestinal Metaplasia. Gastroenterology 2020; 158: 693-702

[9] Pimentel-Nunes P, Libanio D, Marcos-Pinto R et al. Management of epithelial precancerous conditions and lesions in the stomach (MAPS II): European Society of Gastrointestinal Endoscopy (ESGE), European Helicobacter and Microbiota Study Group (EHMSG), European Society of Pathology (ESP), and Sociedade Portuguesa de Endoscopia Digestiva (SPED) guideline update 2019. Endoscopy 2019; 51: 365-388

[10] Oliveira C, Pinheiro H, Figueiredo J et al. Familial gastric cancer: genetic susceptibility, pathology, and implications for management. Lancet Oncol 2015; 16: e60-70

[11] Yaghoobi M, Bijarchi R, Narod SA. Family history and the risk of gastric cancer. Br J Cancer 2010; 102: 237-242

[12] Safaee A, Moghimi-Dehkordi B, Fatemi SR et al. Family history of cancer and risk of gastric cancer in Iran. Asian Pac J Cancer Prev 2011; 12 : 3117-3120

[13] Castano-Rodriguez N, Kaakoush NO, Goh KL et al. The role of TLR2, TLR4 and CD14 genetic polymorphisms in gastric carcinogenesis: a case-control study and meta-analysis. PLoS One 2013; 8: e60327

[14] Castano-Rodriguez N, Kaakoush NO, Pardo AL et al. Genetic polymorphisms in the Toll-like receptor signalling pathway in Helicobacter pylori infection and related gastric cancer. Hum Immunol 2014; 75: 808-815

[15] Castano-Rodriguez N, Kaakoush NO, Goh KL et al. Autophagy in Helicobacter pylori infection and related gastric cancer. Helicobacter 2015; 20: 353-369

[16] Leung WK, Lin SR, Ching JY et al. Factors predicting progression of gastric intestinal metaplasia: results of a randomised trial on Helicobacter pylori eradication. Gut 2004; 53: 1244-1249

[17] Rota M, Pelucchi C, Bertuccio P et al. Alcohol consumption and gastric cancer risk-A pooled analysis within the StoP project consortium. Int J Cancer 2017; 141: 1950-1962
[18] Huang YK, Yu JC, Kang WM et al. Significance of serum pepsinogens as a biomarker for gastric cancer and atrophic gastritis screening: a systematic review and Meta-Analysis. PLoS One 2015; 10: e0142080

[19] Hunt RH, Camilleri M, Crowe SE et al. The stomach in health and disease. Gut 2015; 64: 1650-1668

[20] den Hollander W], Holster IL, den Hoed CM et al. Surveillance of premalignant gastric lesions: a multicentre prospective cohort study from low incidence regions. Gut 2018; 68: 585-593

[21] Yue H, Shan L, Bin L. The significance of OLGA and OLGIM staging systems in the risk assessment of gastric cancer: a systematic review and meta-analysis. Gastric Cancer 2018; 21: 579-587

[22] Tang FB, Li ZX, Wang YM et al. Toll-like receptor 1 and 10 polymorphisms, Helicobacter pylori susceptibility and risk of gastric lesions in a high-risk Chinese population. Infect Genet Evol 2015; 31: 263-269

[23] Zhang K, Zhou B, Wang Y et al. The TLR4 gene polymorphisms and susceptibility to cancer: a systematic review and meta-analysis. Eur J Cancer 2013; 49: 946-954

[24] Ryan BM, Zanetti KA, Robles Al et al. Germline variation in NCF4, an innate immunity gene, is associated with an increased risk of colorectal cancer. Int J Cancer 2014; 134: 1399-1407

[25] Burada F, Ciurea ME, Nicoli R et al. ATG16L1 T300A polymorphism is correlated with gastric cancer susceptibility. Pathol Oncol Res 2016; 22: $317-322$

[26] Ikram MA, Brusselle GGO, Murad SD et al. The Rotterdam Study: 2018 update on objectives, design and main results. Eur J Epidemiol 2017; 32: $807-850$

[27] Peduzzi P, Concato J, Feinstein AR et al. Importance of events per independent variable in proportional hazards regression analysis. II. Accuracy and precision of regression estimates. J Clin Epidemiol 1995; 48: 1503-1510

[28] Zagari RM, Rabitti S, Greenwood DC et al. Systematic review with meta-analysis: diagnostic performance of the combination of pepsinogen, gastrin-17 and anti-Helicobacter pylori antibodies serum assays for the diagnosis of atrophic gastritis. Aliment Pharmacol Ther 2017; 46: 657-667

[29] Wong BC, Lam SK, Wong WM et al. Helicobacter pylori eradication to prevent gastric cancer in a high-risk region of China: a randomized controlled trial. Jama 2004; 291: 187-194

[30] Ma JL, Zhang L, Brown LM et al. Fifteen-year effects of Helicobacter pylori, garlic, and vitamin treatments on gastric cancer incidence and mortality. J Natl Cancer Inst 2012; 104: 488-492

[31] Lin XJ, Wang CP, Liu XD et al. Body mass index and risk of gastric cancer: a meta-analysis. Jpn J Clin Oncol 2014; 44: 783-791

[32] Kneller RW, You WC, Chang YS et al. Cigarette smoking and other risk factors for progression of precancerous stomach lesions. J Natl Cancer Inst 1992; 84: 1261-1266

[33] Okada E, Ukawa S, Nakamura K et al. Demographic and lifestyle factors and survival among patients with esophageal and gastric cancer: The Biobank Japan Project. J Epidemiol 2017; 27: S29-S35

[34] Tramacere I, Negri E, Pelucchi C et al. A meta-analysis on alcohol drinking and gastric cancer risk. Ann Oncol 2012; 23: 28-36

[35] Kim K, Chang Y, Ahn J et al. Smoking and urinary cotinine levels are predictors of increased risk for gastric intestinal metaplasia. Cancer Res 2019; 79: 676-684

[36] Nieminen AA, Kontto J, Puolakkainen P et al. Long-term gastric cancer risk in male smokers with atrophic corpus gastritis. Scand J Gastroenterol 2019; 54: 145-151

[37] Altayar O, Davitkov P, Shah SC et al. AGA technical review on gastric intestinal metaplasia-epidemiology and risk factors. Gastroenterology 2020; 158: 732-744 e716

[38] Leja M, Kupcinskas L, Funka $\mathrm{K}$ et al. Value of gastrin-17 in detecting antral atrophy. Adv Med Sci 2011; 56: 145-150 
[39] Lahner E, Esposito G, Pilozzi E et al. Occurrence of gastric cancer and carcinoids in atrophic gastritis during prospective long-term follow up. Scand J Gastroenterol 2015; 50: 856-865

[40] Reddy KM, Chang JI, Shi JM et al. Risk of gastric cancer among patients with intestinal metaplasia of the stomach in a US integrated health care system. Clin Gastroenterol Hepatol 2016; 14: 1420-1425

[41] Cooney R, Baker J, Brain O et al. NOD2 stimulation induces autophagy in dendritic cells influencing bacterial handling and antigen presentation. Nat Med 2010; 16: 90-97

[42] Mommersteeg MC, Yu J, Peppelenbosch MP et al. Genetic host factors in Helicobacter pylori-induced carcinogenesis: Emerging new paradigms. Biochim Biophys Acta Rev Cancer 2018; 1869: 42-52

[43] Mayerle J, den Hoed CM, Schurmann C et al. Identification of genetic loci associated with Helicobacter pylori serologic status. JAMA 2013; 309: 1912-1920
[44] Dargiene G, Streleckiene G, Skieceviciene J et al. TLR1 and PRKAA1 gene polymorphisms in the development of atrophic gastritis and gastric cancer. J Gastrointestin Liver Dis 2018; 27: 363-369

[45] Loh M, Koh KX, Yeo BH et al. Meta-analysis of genetic polymorphisms and gastric cancer risk: variability in associations according to race. Eur J Cancer 2009; 45: 2562-2568

[46] Lu B, Chen MT, Fan YH et al. Effects of Helicobacter pylori eradication on atrophic gastritis and intestinal metaplasia: a 3-year follow-up study. World J Gastroenterol 2005; 11: 6518-6520

[47] Sung JJ, Lin SR, Ching JY et al. Atrophy and intestinal metaplasia one year after cure of $\mathrm{H}$. pylori infection: a prospective, randomized study. Gastroenterology 2000; 119: 7-14 